\title{
Integrated CCTV Surveillance System for Public Transportation
}

\author{
Dhaval Vibhakar, Suraj Jha, Aditya Kamble, Saurabh Suman
}

\begin{abstract}
The urban center residential district Railway is one in every of the busiest railway stations in Bharat and carries over seven.5 million commuters daily. The railways spreads over $123.78 \mathrm{~km}$ (76.91 mi).The Railways encompass thirty-nine stations. Trains typically begin from and terminate at necessary stations. in line with a survey, the total stats given by the RPF(Railway Police Force) \& GRP(GOVERNMENT RAILWAY POLICE), 2,700 railway commuters killed, over 1,400 whereas crossing tracks up until last and this variety has been increasing daily. This is creating railways a dangerous possibility for travel and transportation. The video closed-circuit television used is irving to be not useful and not updated. To overcome this drawback we tend to area unit creating associate integrated video closed-circuit television for detection of crimes and missed objects and explains during this paper.We area unit exploitation high resolution cameras which might focus and might be simply accustomed establish someone and can also be helpful in dark.

Keywords: Video Surveillance, Facial Recognition, Object Detection, Public Safety and Security.
\end{abstract}

\section{INTRODUCTION}

Railways are a very important a part of human lives. variant folks go past railways as usual. travel through railways is incredibly economical in city however per annum city railways encounters several crimes and thefts. They have an awfully harmful impact on the society. Railway thefts over and over price the loss of human lives, that is that the most fateful Associate in Nursing an irreversible loss.A huge variety of thefts have taken place thanks to lack of security. Through a survey we have a tendency to found that standard makers, we have a tendency to found over one million police investigation cam- eras and over one hundred twenty five thousand police investigation servers exposed to the net. of those devices, ninetieth don't have secure login portals (use HTTP and not HTTPS). Moreover, 8\%open SSH and Telnet ports, have exposed databases like MySQL, and a minimum of one.7\% of those devices are still exposable to the HeartBleed SSL vulnerability discovered in 2012.

Manuscript received on January 09, 2021.

Revised Manuscript received on January 15, 2021.

Manuscript published on February 28, 2021.

* Correspondence Author

Dhaval Vibhakar*, Department of Information Technology, Shree L.R. Tiwari College of Engineering, Thane, India. Email: vibhakar_dhaval@yahoo.com.

Aditya Kamble, Department of Information Technology, Shree L.R. Tiwari College of Engineering, Thane, India. Email: adityakamble619@gmail.com.

Suraj Jha, Department of Information Technology, Shree L.R. Tiwari College of Engineering, Thane, India. Email: surajsambhujha@gmail.com.

Saurabh Suman, Information Technology, Shree L.R. Tiwari College of Engineering, Thane, India. Email: saurabh.suman@slrtce.in

(C) The Authors. Published by Blue Eyes Intelligence Engineering and Sciences Publication (BEIESP). This is an open access article under the CC BY-NC-ND license (http://creativecommons.org/licenses/by-nc-nd/4.0/)
Even well-known makers have vulnerability in their product. As an example, Samsung's CCTV Server has a minimum of eighty two,035 exposed devices, wherever eighty fifth of them use HTTP login portals, the opposite manner around one,604 have ssh ports exposed. Moreover, HikVision, the most important police investigation manufacturer and with banging quantity of shares has additionally exposed devices wherever solely fifty three of them had HTTPS enabled, however with self-verified certificates. This paper presents Associate in Nursing implementation of video police investigation with face recognition. In this paper we have a tendency to ar progressing to use high resolution cameras with inherent South Dakota card for network failure protection. Then alternative vital part video analysis server that is employed to store video with all the opposite elements and description of each topology and composition are going to be explained. Although the IP-based police investigation systems use similar technologies, they're totally different with regards to security. this can be as a result of police investigation systems just about support and enforce our physical security. once comprehend, there's a threat to our physical safety, at home, cooperation, or in an exceedingly national level. As a result, the technology, security motivations dissent from alternative IoTs.

\section{LITERATURE SURVEY}

In survey from 2006-2018 we found out that there are various crimes taken place from local theft to terrorism. In year 2006,Mumbai train bombings were a series of seven bomb blasts on 11 July.In the year 2008,a massive terror attack took place at cst station and many more crimes have taken place in the city. In 2012,woman was brutally assaulted and raped in a moving bus in south Delhi.In 2018 More than 37,000 cases of theft were reported on city railway where commuters lost valuables worth Rs.69 crore. Many deaths have also been taken place to trespassing and unsafe crossing of railway track. The Indian Railway Safety Act, which came into effect in January 1989, it was made to improve human safety through rail by

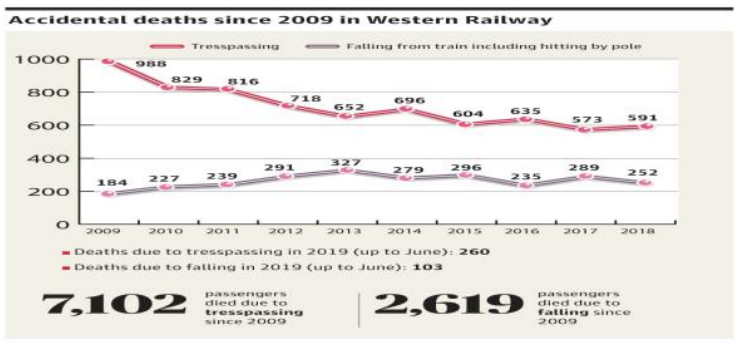

Fig 1:-Reports of deaths since 2009 in Railways.

Published By:

Blue Eyes Intelligence Engineering and Sciences Publication (C) Copvriaht: All riahts reserved. 


\section{Integrated CCTV Surveillance System for Public Transportation}

managing rail safety regulatory frame Work, together with streamlined regulation development and process, and providing railway companies with greater freedom. The present system may not have updated technology and better resolution cameras which increases the work and time of the local railway authority. The figure 1.1 shows a graph analysis of accidental deaths happened in railways since 2009 till 2018.The current video surveillance system has lack of security so anyone can tamper the video easily. Also it does not follow many compliance provided by the Railway authorities. Therefore, in this paper we are proposing an advanced technology with secured server in railway security. This is a help to reduce crimes in the city.

\section{RELATED WORK}

The main objective of video surveillance is to acquire \& process the data so that any suspicious movement can be detected. A lot of research has been done addressing the detection of anomalies in the video data.In [1]Teng Li, Huan Chang, Meng Wang, Bingbing Ni 2015,the proposed system present an the state-of-the-art techniques on crowd analysis which is important at railway station .The problem regarding low resolution video is proposed in[2]V .S.RASMI \& K.R.VINOTHINI 2015.In the proposed paper does not require any training dataset and it also uses background subtraction technique, morphological operations and statistical property standard deviation of the centroids of the objects(blobs) to recognize the occurrence of the unusual events. While [3] Khandu Om 2017, the paper presents an integrated surveillance video system using ip network which is an important aspect of this project. In [4] U.M.Kamthe \& C.G.Patil the proposed system is based on semantic approach,background subtraction. The machine detects the object as living or non-living thing using correlation technique and using motion features \& temporal information are classified as normal or suspicious. In [5]K. Kavikuil and J. Amudha 2018 the proposed paper present deep learning is used to detect anomal behaviour in pre-recorded video. The given model is based on the basic CNN architecture.In [6] 2019 Naor Kalbo, Yisroel Mirsky, Asaf Shabtai, and Yuval Elovici the proposed paper presents a detailed information of ip based surveillance using sql and Iot security, .In [7]Amrutha C.V , C. Jyotsna, Amudha J,2020,the paper presents different combination of artificial intelligence, machine learning and deep learning pitched into the system

\section{PROPOSED SYSTEM METHODOLOGY}

Unlike ancient cctv video closed-circuit television,we designed the system exploitation IP-based video police investigation.The video closed-circuit television depends upon the users wants. The methodology involves assets like observation , Topology , Protection , Host , social control.

Assets:-

Cameras - The video capturing is finished by the camera . There area unit varied sorts of IP- Cameras, every having its capabilities and vulnerabilities. Some informatics cameras give web-based interfaces whereas others hook up with a server within the cloud for configuration. Most camera act as an internet servers which offer video content to licensed purchasers. Three sorts of cameras are going to be majorly used Box/Bullet/PTZ Cameras with high resolution and veri-focal lenses. The cameras have intrinsical SD-Card for network failure detention protection thus whenever any network failure happens the cameras area unit capable of storing the video police investigation throughout the time of network failure and so it's hold on within the server once the network is recovered.

VMS - A Video Management System (VMS)or Video Management Server may be a element of security camera system.It is a package element of network video recorder and digital video recorder.

The VMS tends to produce be a lot of refined however additionally provides a lot of capabilities.The options to be thought-about of VMS area unit like video-analytics, Integration with third-party systems, like access management, building automation, alarm management, video analytics and a lot of,Camera management (Pan,Tilt,Zoom),view multiple video promptly,Multichannel playback, that permits users to play recorded video from many cameras simultaneously,Fail-over capability that allows continuing recording if the first server goes down.

NVR- Media Server A Network Video recorder is specialised laptop package that records video in digital format on the server. NVR systems takes proof and store video on the network it's enforced. These systems work with a complicated kind of camera, known as informatics cameras.A sixty four channel NVR supports upto sixty four informatics Cameras, it will each work alone as a recorder and work with different device to builds a comprehensive closed-circuit television.

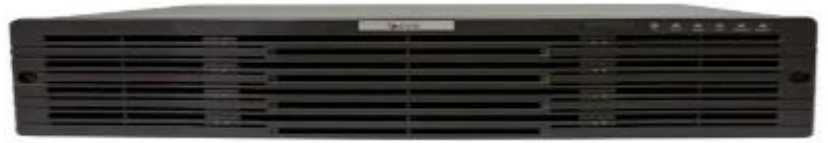

Fig 2:64 ch NVR.

The NVR provides a playback potency with a speed starting from traditional to $16 \mathrm{x}$ speed.

Video Content:-The video feeds that area unit being recorded or that area unit archived for later viewing.

Permission - The user names, passwords, cookies, and authentication tokens accustomed gain access to the DVR, cameras, and routers. The credentials area unit accustomed au- thenticate users and confirm access permissions of video , configurations of devices, and different devices.

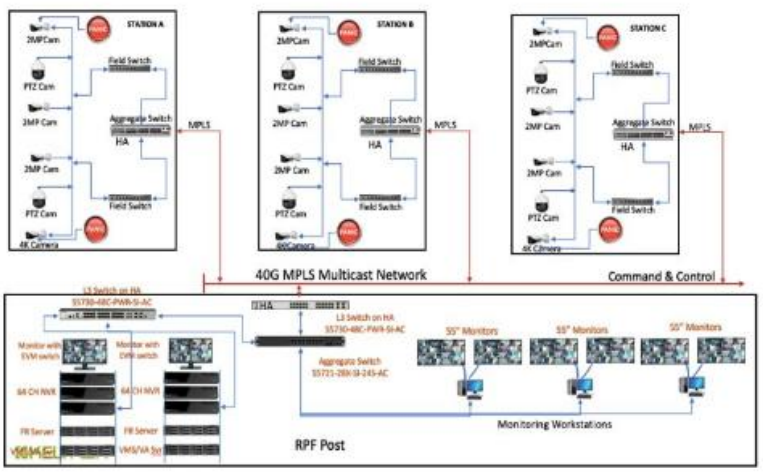

Fig 2:-Basic Setup diagram

Published By:

Blue Eyes Intelligence Engineering and Sciences Publication

DOI:10.35940/ijeat.C2157.0210321

Journal Website: www.ijeat.org

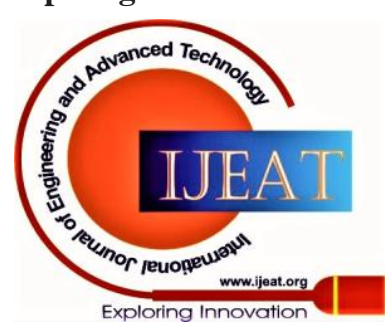


Interface POE-Power Over LAN lets LAN cables provide power to network devices over the present knowledge affiliation. Category five cable or class 5 cable may be used for writers.

MPLS: Multicast Network-Multiprotocol Label switch (MPLS) VPNs may be a packet forwarding methodology exploitation labels to form knowledge Forwarding selections. MPLS allows scalable VPNs, end-to-end QoS, and different informatics services that enable economical utilization of existing networks with easier configuration and management and faster fault correction.

MPLS may be accustomed produce forwarding tables for any underlying protocol.MPLS routers establish a label-switched path (LSP), a preset path to route traffic in associate degree MPLS network, supported the factors within the FEC. MPLS forwarding will occur solely once LSP has been established. LSPs area unit simplex which implies that come back traffic is shipped over a special LSP.Here a 10G mpls circuit is employed for WAN and from flexibility and ports.
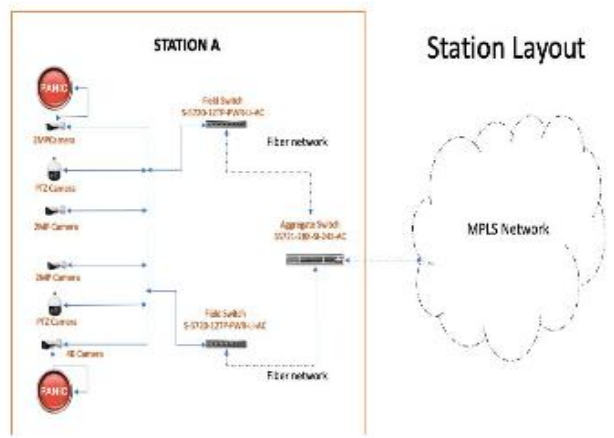

Fig 3:Basic station platform Layout.

Topology-The IP-based closed-circuit television topology may be delineated by its layout.Layout refers as to whether the cameras area unit situated anyplace within the world or physically situated in one space.Star topology every camera within the network is connected to a field switch exploitation associate degree combination switch. The complete network is connected to every different via wired cables (Ethernet,cat6,Stp cables,etc) or wireless(e.g Wi-fi) or each.

Communication-This refers to a way to video feed is transported within the system.With digital signal being transferred with the assistance of NVR.With digital methodology the video is n sent as a packet stream to NVR via IPv4 and IPv6 protocols and so hold on and viewed .A common approach is to compress the video streams with H.265 codec and so send it over the network with a true time protocol like RTP and UDP .Facial Recognition identity verification identity verification a engineering being employed during a sort of applications that identifies human faces in digital pictures.The types of identity verification system utilized in this project area unit human identification at a distance , Thermal cameras to examine the temperature of a person's body. As the system is unsupervised it adapts to learning day-by day.The system has associate degree proactive approach at the drawing board.

ONVIF Protocol-Open Network Video Interface Forum.It is is each a worldwide forum and a worldwide protocol that allows completely different|completely different $\}$ police investigation and security devices from different makers to figure along seamlessly.ONVIF presently has six profiles for security devices.For video police investigation 2 main profiles area unit G and S.An ONVIF Profile G device (e.g., associate degree informatics network camera or video encoder) is one which will store video-input knowledge over associate degree informatics network or on the device itself. A Profile G consumer (e.g., a video management software) is one which is able to organize,request, associate degreed management recording of video knowledge over an informatics network from a Profile G designed device. Profile $G$ additionally includes support for receiving audio and data stream if the consumer supports those options.An ONVIF Profile S device (e.g., associate degree informatics network camera or video encoder) is one that transmits video knowledge over associate degree informatics network to a Profile S designed consumer. A Profile S consumer is one which will construct, request, associate degreed management video streaming over an informatics network from a Profile $S$ designed device. Profile S additionally covers ONVIF specifications for movement of cameras,sound-input, and varied different specification outputs for designed devices and purchasers that support such protocol.

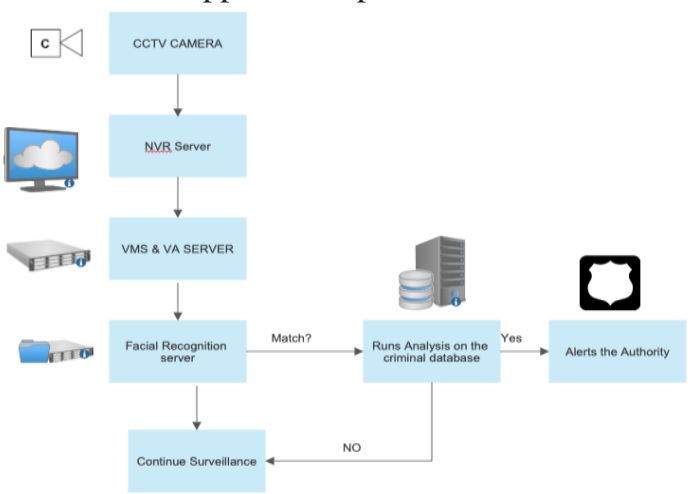

Fig 4:-Flow Diagram of the proposed system

The above figure explains the flow diagram of the whole proposed system as follows:

Step 1:The cctv camera captures the person's face and sends it to the Network Video Recorder System.

Step 2:The Network Video Recorder system records the video and sends its to the video management system and video analytics for surveillance purposes.

Step 3:-The Vms Send the video to different monitors and then to the facial recognition server.

Step 4:The facial recognition server analyses the particular face using different algorithms and matches it with the database provided by the authorities and if there is a match the authority will be alerted or else it will continue to record the surveillance.

\section{RESULTS}

The facial detection and object detection in the video data is a challenging task. It has a number of difficulties such as complexity of scene, illumination of light, camera angle etc. Also the definition of the suspect activity is scene/place dependent. For eg,the complete detection of man is shown in the video.

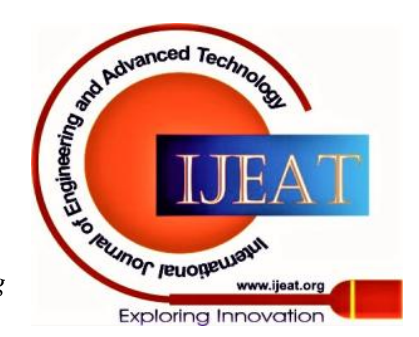




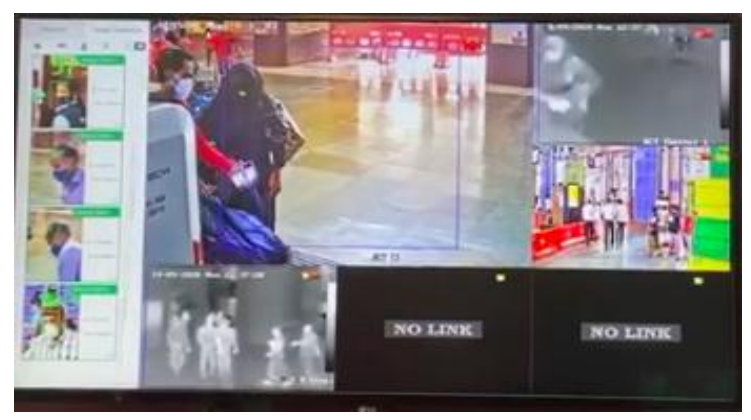

VI. CONCLUSION

In this survey, we have reviewed the security of integrated video surveillance systems. We have presented an overview of these systems, listed the system's assets. The results of the facial recognition is accurate and also reactive approach as the system keeps learning the data set. We can also implement this system in other transport stations like bus station, taxi station. We can also implement cloud based systems in our project. Finally, we provided a brief summary of best practices and security solutions which can be used to increase the security. The system will help to ensure that any person is not of any kind of threat to the society. We hope that this article will help to enhance security and create a crime free society.

\section{ACKNOWLEDGMENT}

This project was conducted under the guidance and funded by Insight business machine Pvt. Ltd.

\section{REFERENCES}

1. 2015,Teng Li, Huan Chang, Meng Wang, Bingbing Ni, Richang Hong,and Shuicheng Yan, Senior Member, "Crowded Scene Analysis: A Survey”,IEEE Transactions On Circuits And Systems For Video Technology, VOL. 25, NO. 3, MARCH 2015.

2. 2015, V.S.Rasmi \& K.R.Vinthoni "Real Time Unusual Event Detection Using Video Surveillance System For Enhancing Security".

3. 2016,Shalince Dominic,Mahesh Mohan,Aparna C,Ajeesh M S,Aswin S Nath,Anil Antony "A REVIEW OF FACE DETECTION SYSTEM"International Conference on Electrical, Electronics, and Optimization Techniques (ICEEOT).

4. 2017, Khandu Om "Integrated Access Control and Video Surveillance".

5. 2018,U.M.Kamthe \& C.G.Patil "Suspicious Activity Recognition in Video Surveillance System”,2018 IEEE.

6. 2018,Shivam Bachhety,Ramneek Singhal, Kshitiz Rawat, Kunal Joshi, Rachna Jain "Crime Detection Using Text Recognition and Face Recognition'International Journal of Pure and Applied Mathematics Volume 119 No. 15 2018, 2797-2807.

7. 2019, K. Kavikuil and J. Amudha "Leveraging Deep Learning for Anomaly Detection in Video Surveillance".

8. 2019,Naor Kalbo, Yisroel Mirsky, Asaf Shabtai, and Yuval Elovic "The Security of IP-based Video Surveillance Systems",IEEE,Oct 2019

9. 2020,Amrutha C.V, C. Jyotsna, Amudha J,Amrita Vishwa Vidyapeetham, "Deep Learning Approach for Suspicious Activity Detection from Surveillance Video", Proceedings of the Second International Conference on Innovative Mechanisms for Industry Applications (ICIMIA 2020) IEEE Xplore Part Number: CFP20K58-ART; ISBN: 978-1-7281-4167-1

10. 2019,Apoorva.P,Impana.H.C,Siri.S.L,Varshitha.M.R,Ramesh.B“AU TOMATED CRIMINAL IDENTIFICATION BY FACE RECOGNITION USING OPEN COMPUTER VISION

11. CLASSIFIERS" Proceedings of the Third International Conference on Computing Methodologies and Communication (ICCMC 2019) IEEE Xplore Part Number: CFP19K25-ART; ISBN: 978-1-5386-7808-4

\section{AUTHORS PROFILE}

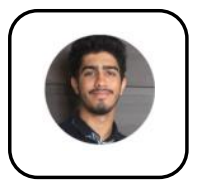

Dhaval Vibhakar is a BE-IT student at Shree L.R. Tiwari College of Engineering, Mira Road and is expected to graduate in May 2021. His domain of interest and research are Cloud Computing, Software development and Web development. Dhaval has done certification on courses such as web development and cloud computing. To aggrandize to his portfolio Dhaval is applying for Masters in computer science in fall 2022.He has also worked on various projects like smart car parking system in IOT. Dhaval aims to become an excellent programmer and learn different technologies to find better and innovative solutions.

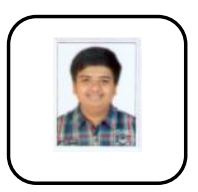

Aditya Kamble is a BE-IT student at Shree L.R Tiwari College of Engineering, Mira Road and is expected to graduate in May 2021. .His domain of interest and research are Machine learning and Database management. He has also worked on various projects like Homework Writing Machine in IOT. Aditya aims to learn about detail in artificial intelligence which will help to make innovative products.

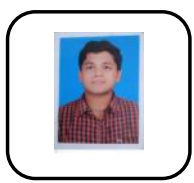

Suraj Jha Kamble is a BE-IT student at Shree L.R. Tiwari College of Engineering, Mira Road and is expected to graduate in May 2021. His domain of interest and research are Machine learning and Python Programming .He has also worked on various projects like Health Monitroing System in IOT. Suraj aims to learn about detail in advanced python programming which will help to make innovative products.

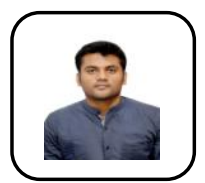

Saurabh Suman is an Assistant Professor in the department of Information Technology at Shree L.R. Tiwari College of Engineering. His educational background includes BTech and MTech in Computer Engineering. Currently, he is pursuing his PhD. Prof. Saurabh Suman has a teaching experience of 10 years. His research area is IoT and Computer Networks. Not only has he acquired numerous certifications in the above-mentioned fields but also, he has organized several competitions, seminar and webinars to disseminate the knowledge of IoT and Computer Networks.

Published By:

Blue Eyes Intelligence Engineering and Sciences Publication

DOI:10.35940/ijeat.C2157.0210321

Journal Website: www.ijeat.org

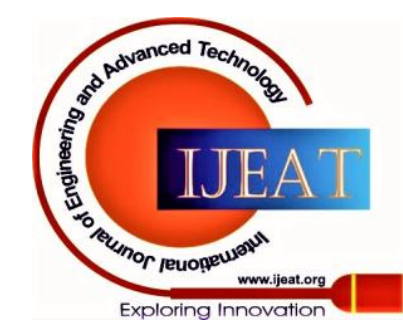

